\title{
W[1]-hardness of the $k$-center problem parameterized by the skeleton dimension
}

\author{
Johannes Blum ${ }^{1}$
}

Accepted: 2 August 2021 / Published online: 12 August 2021

(C) The Author(s) 2021

\begin{abstract}
We study the $k$-CENTER problem, where the input is a graph $G=(V, E)$ with positive edge weights and an integer $k$, and the goal is to select $k$ center vertices $C \subseteq V$ such that the maximum distance from any vertex to the closest center vertex is minimized. In general, this problem is NP-hard and cannot be approximated within a factor less than 2. Typical applications of the $k$-CENTER problem can be found in logistics or urban planning and hence, it is natural to study the problem on transportation networks. Common characterizations of such networks are graphs that are (almost) planar or have low doubling dimension, highway dimension or skeleton dimension. It was shown by Feldmann and Marx that $k$-CENTER is W[1]-hard on planar graphs of constant doubling dimension when parameterized by the number of centers $k$, the highway dimension $h d$ and the pathwidth pw (Feldmann and Marx 2020). We extend their result and show that even if we additionally parameterize by the skeleton dimension $\kappa$, the $k$-CENTER problem remains W[1]-hard. Moreover, we prove that under the Exponential Time Hypothesis there is no exact algorithm for $k$-CENTER that has runtime $f(k, h d, p w, \kappa) \cdot|V|^{o(p w+\kappa+\sqrt{k+h d})}$ for any computable function $f$.
\end{abstract}

Keywords $k$-Center $\cdot$ Skeleton dimension $\cdot$ Highway dimension $\cdot$ Parameterized complexity

\section{Introduction}

The $k$-CENTER problem consists of the following task: Given a graph $G=(V, E)$ with positive edge weights $\ell: E \rightarrow \mathbb{Q}^{+}$and some positive integer $k$, choose $k$ center vertices $C \subseteq V$ that minimize the maximum distance from any vertex of the graph to the closest center. Formally, denote the shortest path distances in the graph $G$ by dist: $V^{2} \rightarrow \mathbb{Q}^{+}$and let $B_{r}(v)=\{w \in V \mid \operatorname{dist}(v, w) \leq r\}$ be the ball of radius $r$

\footnotetext{
$凶$ Johannes Blum

johannes.blum@uni-konstanz.de

1 Universität Konstanz, Universitätsstr. 10, 78457 Konstanz, Germany
} 
around $v$. We define the cost of a solution $C \subseteq V$ as the smallest radius $r \geq 0$ such that $V=\bigcup_{v \in C} B_{r}(v)$ and the goal is to find a solution of size at most $k$ that has minimum cost.

As $k$ - CENTER is a generalization of the DOMINATING SET problem, it is NP-hard on general graphs. Therefore, approximation algorithms and fixed-parameter algorithms for $k$-CENTER have been studied extensively in different settings. Due to Hochbaum and Shmoys (1986) there is a 2-approximation algorithm, i.e., an efficient algorithm that computes a solution which deviates from the optimum at most by a factor of 2 . This factor is tight, as for any $\epsilon>0$, it is NP-hard to compute a $(2-\epsilon)$-approximation, even when considering planar graphs (Plesník 1980) or geometric graphs using $L_{1}$ or $L_{\infty}$-distances (Feder and Greene 1988).

Still, the $k$-CENTER problem has common applications in areas like logistics or urban planning. Imagine for example that we want to place a limited number of warehouses, hospitals or police stations in some city. However, there is only a budget to build $k$ such facilities and the task is to find $k$ locations such that the distance from any point to the closest facility is a small as possible. Thus, it is very reasonable to study $k$-CENTER on transportation networks. Such networks are commonly modeled as planar graphs or graphs that have low doubling dimension, highway dimension, or skeleton dimension. For formal definitions of these parameters, see Sect. 2.

It is usually assumed that in graphs that model transportation networks, the mentioned parameters are bounded by $\mathcal{O}$ (polylog $|V|)$ or $\mathcal{O}(\sqrt{|V|})$. It was shown that on graphs of maximum degree $\Delta$ and highway dimension $h d$, the skeleton dimension is at most $(\Delta+1) \cdot h d$ (Kosowski and Viennot 2017). The relationship between highway dimension $h d$ and skeleton dimension $\kappa$ was also evaluated experimentally on several real-world road networks and it turned out that $\kappa \ll h d$ (Blum and Storandt 2018). Moreover, it was conjectured that on road networks the skeleton dimension is a constant whereas the highway dimension grows faster than $\mathcal{O}$ (polylog|V|).

Still, graphs of low highway dimension or skeleton dimension do not allow better approximation factors than 2. In particular, it was shown that for any $\epsilon>0$, it is NP-hard to compute a $(2-\epsilon)$-approximation for graphs of highway dimension $h d \epsilon$ $\mathcal{O}\left(\log ^{2}|V|\right)\left(\right.$ Feldmann 2019) or skeleton dimension $\kappa \in \mathcal{O}\left(\log ^{2}|V|\right)$ (Blum 2019).

Another way of dealing with NP-hard problem is parameterization. The idea is to capture the complexity of the problem through some parameter $p$ which is independent of the problem size $n$. A fixed-parameter algorithm computes an exact solution in time $f(p) \cdot n^{\mathcal{O}(1)}$ where $f$ is a computable function and $p$ a parameter. If a certain problem admits such an fixed-parameter algorithm, we call it also fixed-parameter tractable (FPT).

When studying the fixed-parameter tractability of $k$ - CENTER, a very natural parameter is the number of centers $k$. However, $k$ - CENTER is W[2]-hard for parameter $k$, which stems from the DOMINATING SET problem, and hence $k$-CENTER in not fixedparameter tractable for parameter $k$ unless FPT $=\mathrm{W}[2]$.

The fixed-parameter tractability of $k$ - CENTER on transportation networks was studied by Feldmann and Marx (2020). They showed that even if the input is restricted to planar graphs of constant doubling dimension, the problem is W[1]-hard when the parameter combines $k$, the highway dimension $h d$ and the pathwidth $p w$. Moreover, they proved that under the Exponential Time Hypothesis (ETH) there is no exact algo- 
rithm with runtime $f(k, p w, h d) \cdot|V|^{o(p w+\sqrt{k+h d})}$. In the present paper we extend this result and show that one can additionally parameterize by the skeleton dimension $\kappa$ without affecting W[1]-hardness. Formally, we show the following theorem.

Theorem 1 On planar graphs of constant doubling dimension, the $k$ - CENTER problem is W[1]-hardfor the combined parameter $(k, p w, h d, \kappa)$ where pw is the pathwidth, hd the highway dimension and $\kappa$ the skeleton dimension of the input graph. Assuming ETH there is no $f(k, p w, h d, \kappa) \cdot|V|^{o(p w+\kappa+\sqrt{k+h d})}$ time algorithm ${ }^{1}$ for any computable function $f$.

In the reduction of Feldmann and Marx, the maximum degree $\Delta$ of the produced graph can be quadratic in the input size. As it holds that $\Delta \leq \kappa$, we cannot conclude any parameterized hardness for the skeleton dimension. Our new construction yields a graph of constant maximum degree, which enables us to bound the skeleton dimension as well as the highway dimension and the pathwidth.

Experiments performed by Blum and Storandt (2018) indicate that in real-world road networks, the skeleton dimension $\kappa$ is significantly smaller than the highway dimension, which motivates the use of $\kappa$ as a parameter. Note that in general, the parameters $p w, h d$ and $\kappa$ are incomparable (Blum 2019). However, our main result shows that combining all these parameters and the number of centers $k$ does not allow a fixed-parameter algorithm unless FPT $=\mathrm{W}[1]$.

Still, if a problem is hard to approximate and does not allow any fixed-parameter algorithm, it can help to combine the two paradigms. For the $k$-CENTER problem, parameterization indeed allows to achieve better approximation factors than 2 . For instance, there is a so called efficient parameterized approximation scheme (EPAS) parameterized by $k$ and the highway dimension $h d$, i.e., an algorithm computing a $(1+\epsilon)$-approximation for any $\epsilon>0$ in time $f(k, h d, \epsilon) \cdot n^{\mathcal{O}(1)}$ for some computable function $f$ (Becker et al. 2018). Moreover, there is an EPAS parameterized by $k$ and the doubling dimension (Feldmann and Marx 2020). As any graph of skeleton dimension $\kappa$ has doubling dimension at most $\log _{2}(2 \kappa+1)$ (Kosowski and Viennot 2017), this also yields an EPAS for parameter $(k, \kappa)$. Theorem 1 implies that approximation is indeed necessary when parameterizing by any of $k, h d, \kappa$ and $p w$.

\section{Preliminaries}

For $n \in \mathbb{N}$, let $[n]=\{1, \ldots, n\}$. Addition modulo 4 is denoted by $\boxplus$ and the lexicographic order on $\mathbb{N} \times \mathbb{N}$ is denoted by $\leq$, i.e., $(a, b) \leq\left(a^{\prime}, b^{\prime}\right)$ if and only if $a<a^{\prime}$ or $a=a^{\prime}$ and $b \leq b^{\prime}$.

In a graph $\bar{G}=(V, E)$ let $\pi(s, t)$ be the shortest $s$ - $t$ path and let $\ell(P)$ be the length of a path $P$. The concatenation of two paths $P$ and $P^{\prime}$ is denoted by $P \circ P^{\prime}$.

A graph $G$ is planar if it can be embedded into the plane without crossing edges, and $d$-doubling if for any $r>0$, any ball $B_{2 r}(v)$ of radius $2 r$ in $G$ is contained in the union of $d$ balls of radius $r$. If $d$ is the smallest integer such that $G$ is $d$-doubling, the graph $G$ has doubling dimension $\log _{2} d$.

\footnotetext{
${ }^{1}$ Here $o(p w+\kappa+\sqrt{k+h d})$ stands for $g(p w+\kappa+\sqrt{k+h d})$. where $g$ is a function with $g(x) \in o(x)$.
} 
For the highway dimension several slightly different definitions can be found in the literature (Abraham et al. 2010, 2011, 2016). Here we use the original one given by Abraham et al. (2010). Note that our results also hold when using the definition from (Abraham et al. 2011). For a discussion on the different definitions, we refer to (Blum 2019).

Definition 1 The highway dimension of a graph $G$ is the smallest integer $h d$ such that for any radius $r$ and any vertex $v$ there is a hitting set $S \subseteq B_{4 r}(v)$ of size $h d$ for the set of all shortest paths $\pi$ satisfying $|\pi|>r$ and $\pi \subseteq B_{4 r}(v)$.

To define the skeleton dimension, which was introduced in (Kosowski and Viennot 2017), we need to consider the geometric realization $\tilde{G}$ of a graph $G$. Intuitively, $\tilde{G}$ is a continuous version of $G$ where every edge is subdivided into infinitely many infinitely short edges. For a vertex $s \in V$, let $T_{s}$ be the shortest path tree of $s$. We assume that in $G$ every shortest path is unique, which can be achieved, e.g., by slightly perturbing the edge weights, and it follows that $T_{S}$ is also unique. The skeleton $T_{S}^{*}$ of the shortest path tree $T_{S}$ is defined as the subtree of $\tilde{T}_{S}$ induced by all vertices $v$, for which there is some vertex $w$ such that $v$ is contained in $\pi(s, w)$ and moreover, we have $\operatorname{dist}(s, v) \leq 2 \cdot \operatorname{dist}(v, w)$. Intuitively, we obtain the skeleton $T_{s}^{*}$ by cutting off the last third of every branch of the shortest path tree $T_{s}$.

Definition 2 For a shortest path tree skeleton $T_{s}^{*}=\left(V^{*}, E^{*}\right)$ in a graph $G$ and a radius $r>0$, let $\operatorname{Cut}_{r}(s):=\left\{v \in V^{*} \mid \operatorname{dist}(s, v)=r\right\}$. The skeleton dimension of a graph $G$ is $\kappa:=\max _{s, r}\left|\operatorname{Cut}_{r}(s)\right|$.

We conclude this section with a definition of the pathwidth.

Definition 3 A path decomposition of a graph $G=(V, E)$ is a sequence $\left(X_{1}, \ldots, X_{r}\right)$ where every $X_{i}$ (also called bag) is a subset of $V$ and the following properties are satisfied:

(1) $\bigcup_{i=1}^{r} X_{i}=V$,

(2) for every edge $\{u, v\} \in E$ there is a bag $X_{i}$ containing both $u$ and $v$, and

(3) for every three indices $i \leq j \leq$ we have $X_{i} \cap X_{k} \subseteq X_{j}$.

The width of a path decomposition is the size of the largest bag minus one, i.e. $\max _{i=1}^{r}\left(\left|X_{i}\right|-1\right)$. The pathwidth $p w$ of a graph $G=(V, E)$ is defined as the minimum width of all path decompositions of $G$.

\section{The reduction}

Following the idea of Feldmann and Marx (2020), who showed that on planar graphs of constant doubling dimension, $k$ - CENTER is W[1]-hard for parameter $(k, p w, h d)$, we present a reduction from the GRID TILING WITH INEQUALITY $\left(\mathrm{GT}_{\leq}\right)$problem. This problem asks the following question: Given $\chi^{2}$ sets $S_{i, j} \subseteq[n]^{2}$ of pairs of integers, where $(i, j) \in[\chi]^{2}$, is it possible to choose one pair $s_{i, j} \in S_{i, j}$ from every set, such that 
- if $s_{i, j}=(a, b)$ and $s_{i+1, j}=\left(a^{\prime}, b^{\prime}\right)$ we have $a \leq a^{\prime}$, and

- if $s_{i, j}=(a, b)$ and $s_{i, j+1}=\left(a^{\prime}, b^{\prime}\right)$ we have $b \leq b^{\prime}$.

It is known that the $\mathrm{GT}_{\leq}$problem is W[1]-hard for parameter $\chi$ and, unless the Exponential Time Hypothesis (ETH) fails, it has no $f(\chi) \cdot n^{o(\chi)}$ time algorithm for any computable $f$ (Cygan et al. 2015).

\subsection{The reduction of Feldmann and Marx}

Given some instance $\mathcal{I}$ of $\mathrm{GT}_{\leq}$, the reduction from (Feldmann and Marx 2020) produces the following graph $H_{\mathcal{I}}$. For any of the $\chi^{2}$ sets $S_{i, j}$, the graph $H_{\mathcal{I}}$ contains a gadget $H_{i, j}$ that consists of a cycle $O_{i, j}=v_{1} v_{2} \ldots v_{16 n^{2}+4} v_{1}$ and five additional vertices $x_{i, j}^{1}, x_{i, j}^{2}, x_{i, j}^{3}, x_{i, j}^{4}$, and $y_{i, j}$. For all $i, j$, every edge of the cycle $O_{i, j}$ has unit length and the vertex $y_{i, j}$ is connected to $O_{i, j}$ via edges to $v_{1}, v_{4 n^{2}+2}, v_{8 n^{2}+3}$ and $v_{12 n^{2}+4}$, which all have length $2 n^{2}+1$. Moreover, for every pair $(a, b) \in S_{i, j}$ and $\tau=(a-1) \cdot n+b$, the gadget $H_{i, j}$ contains the four edges

$-\left\{x_{i, j}^{1}, v_{\tau}\right\}$ of length $2 n^{2}-\frac{a}{n+1}$,

$-\left\{x_{i, j}^{2}, v_{\tau+4 n^{2}+1}\right\}$ of length $2 n^{2}+\frac{b}{n+1}-1$,

$-\left\{x_{i, j}^{3}, v_{\tau+8 n^{2}+2}\right\}$ of length $2 n^{2}+\frac{a}{n+1}-1$, and

$-\left\{x_{i, j}^{4}, v_{\tau+12 n^{2}+3}\right\}$ of length $2 n^{2}-\frac{b}{n+1}$.

Finally, the individual gadgets are connected in a grid-like fashion, which means that there is a path from $x_{i, j}^{2}$ to $x_{i, j+1}^{4}$ and from $x_{i, j}^{3}$ to $x_{i+1, j}^{1}$. Each of these paths has length 1 and consists of $n+2$ edges of length $\frac{1}{n+2}$.

Feldmann and Marx showed that the given $\mathrm{GT}_{\leq}$instance $\mathcal{I}$ has a solution if and only if the $k$-CENTER problem in the graph $H_{\mathcal{I}}$ has a solution of cost $2 n^{2}$ using $k=5 \chi^{2}$ centers. Moreover, the graph $H_{\mathcal{I}}$ is planar and has doubling dimension $\mathcal{O}(1)$, highway dimension $\mathcal{O}\left(\chi^{2}\right)$ and pathwidth $\mathcal{O}(\chi)$. Observe that the degree of any vertex $x_{i, j}^{h}$ is $\left|S_{i, j}\right|$. This means that the skeleton dimension of $H_{\mathcal{I}}$ might be as large as $\Omega\left(n^{2}\right)$, as the maximum degree of $H_{\mathcal{I}}$ is a lower bound on its skeleton dimension. We show now how to construct a graph $G_{\mathcal{I}}$ that resembles $H_{\mathcal{I}}$, but has skeleton dimension $\mathcal{O}(\chi)$ and fulfills the other mentioned properties.

\subsection{Our construction}

We assume that in the given $\mathrm{GT}_{\leq}$-instance, for all $(i, j) \in[\chi]^{2}$ and every $b \in[n]$, there is some $a \in[n]$ such that $(a, b) \in S_{i, j}$. This is a valid assumption, as from an instance $\mathcal{I}$ of ordinary $\mathrm{GT}_{\leq}$, we can construct the following instance $\mathcal{I}^{\prime}$. For $i \in[\chi-1]$ and $j \in[\chi]$ we add the pairs $\{(n+\chi-i, b) \mid b \in[n]\}$ to $S_{i, j}$. Moreover, we add the pairs $\{(0, b) \mid b \in[n]\}$ to every $S_{\chi, j}$. Clearly, every solution for $\mathcal{I}$ is also a solution for $\mathcal{I}^{\prime}$. Consider now a solution for $\mathcal{I}^{\prime}$. For $(i, j) \in[\chi-1] \times[\chi]$ we cannot choose a "dummy" pair $s_{i, j}=(n+\chi-i, b)$, as there is no $\left(a^{\prime}, b^{\prime}\right) \in S_{i+1, j}$ such that $a^{\prime} \geq n+\chi-i$. Moreover, it is not possible to choose $s_{\chi, j}=\left(0, b^{\prime}\right)$ as $S_{\chi-1, j}$ contains no pair $(a, b)$ satisfying $a \leq 0$. Hence, $\mathcal{I}$ has a solution if and only if $\mathcal{I}^{\prime}$ has a solution. 


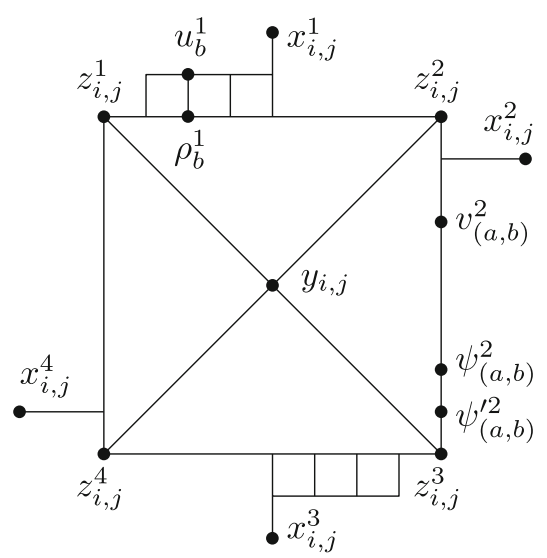

(a) A gadget $G_{i, j}$.

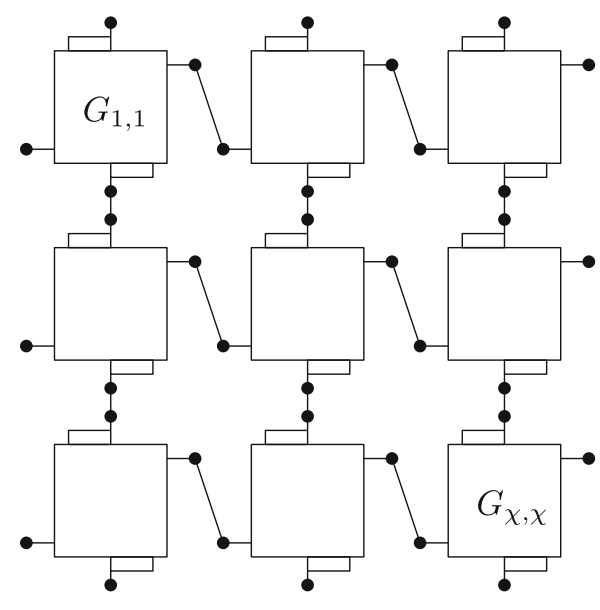

(b) The graph $G_{\mathcal{I}}$.

Fig. 1 A single gadget $G_{i, j}$ and the whole graph $G_{\mathcal{I}}$

Given a $\mathrm{GT}_{\leq}$-instance $\mathcal{I}$ we construct the following graph $G_{\mathcal{I}}$ (cf. Fig. 1). Like in (Feldmann and Marx 2020), we create a gadget $G_{i, j}$ for every set $S_{i, j}$. Any $G_{i, j}$ contains a cycle $O_{i, j}$, which initially consists of four edges that have length $2^{n+2}+1 / n$. Denote the four vertices of the cycle $O_{i, j}$ by $z_{i, j}^{1}, \ldots, z_{i, j}^{4}$ and for $h \in$ [4] let $O_{i, j}^{h}=$ $\pi\left(z_{i, j}^{h}, z_{i, j}^{h \boxplus 1}\right)$. Now, for any pair $(a, b) \in S_{i, j}$ and any $h \in[4]$ we insert a vertex $v_{(a, b)}^{h}$ into the path $O_{i, j}^{h}$ and place it such that its distance to $z_{i, j}^{h}$ is

$$
d_{(a, b)}:=2^{b}-1+\frac{a}{n}
$$

It follows that the distance between $v_{(a, b)}^{h}$ and $v_{(a, b)}^{h \boxplus 1}$ is $2^{n+2}+1 / n$. Moreover, for $\left(a^{\prime}, b^{\prime}\right) \leq(a, b)$, the distance from $v_{\left(a^{\prime}, b^{\prime}\right)}^{h}$ to $v_{(a, b)}^{h}$ is $2^{b}-2^{b^{\prime}}+\left(a-a^{\prime}\right) / n$.

Additionally, for any pair $(a, b) \stackrel{\in}{\in} S_{i, j}$ and any $h \in$ [4], we insert two vertices $\psi_{(a, b)}^{h}$ and $\psi_{(a, b)}^{\prime h}$ into the path $O_{i, j}^{h}$ such that their distances from $v_{(a, b)}^{h}$ are $2^{n+1}$ and $2^{n+1}+1 / n$, respectively. This implies that

$$
\begin{aligned}
& \operatorname{dist}\left(v_{(a, b)}^{h}, \psi_{(a, b)}^{h}\right)=\operatorname{dist}\left(\psi_{(a, b)}^{\prime h}, v_{(a, b)}^{h \boxplus 1}\right)=2^{n+1} \text { and } \\
& \operatorname{dist}\left(v_{(a, b)}^{h}, \psi_{(a, b)}^{\prime h}\right)=\operatorname{dist}\left(\psi_{(a, b)}^{h}, v_{(a, b)}^{h \boxplus 1}\right)=2^{n+1}+\frac{1}{n} .
\end{aligned}
$$

Any gadget $G_{i, j}$ also contains a central vertex $y_{i, j}$ that is connected to each $z_{i, j}^{h}$ through an edge of length $2^{n+1}+1$. Finally, we add four vertices $x_{i, j}^{1}, \ldots, x_{i, j}^{4}$ to every gadget $G_{i, j}$, through which we will connect the individual gadgets. For $(a, b) \in$ $S_{i, j}$ and $h \in[4]$ denote the distance between $v_{(a, b)}^{h}$ and $x_{i, j}^{h}$ by $d_{(a, b)}^{h}$. The idea of our reduction is that we attach every $x_{i, j}^{h}$ to the cycle $O_{i, j}$ such that for every pair 
$(a, b) \in S_{i, j}$ and $h \in\{1,3\}$, the distance $d_{(a, b)}^{h}$ reflects the value of $b$, whereas for $h \in\{2,4\}$, the distance $d_{(a, b)}^{h}$ reflects the value of $a$.

For the latter, we simply add an edge between $x_{i, j}^{2}$ and the vertex $v_{\left(a^{*}, b^{*}\right)}^{2}$ where $\left(a^{*}, b^{*}\right)=\min S_{i, j} \cdot{ }^{2}$ The length of this edge is chosen as

$$
d_{\left(a^{*}, b^{*}\right)}^{2}:=2^{n}+1+d_{\left(a^{*}, b^{*}\right)}=2^{n}+2^{b^{*}}+\frac{a^{*}}{n} .
$$

Similarly we add the edge $\left\{x_{i, j}^{4}, v_{\left(a^{*}, b^{*}\right)}^{4}\right\}$ and set its length to

$$
d_{\left(a^{*}, b^{*}\right)}^{4}:=2^{n+1}-d_{\left(a^{*}, b^{*}\right)}=2^{n+1}+1-2^{b^{*}}-\frac{a^{*}}{n} .
$$

It follows that for all $(a, b) \in S_{i, j}$ we have

$$
\begin{aligned}
& d_{(a, b)}^{2}=d_{\left(a^{*}, b^{*}\right)}^{2}+d_{(a, b)}-d_{\left(a^{*}, b^{*}\right)}=2^{n}+2^{b}+\frac{a}{n} \text { and } \\
& d_{(a, b)}^{4}=d_{\left(a^{*}, b^{*}\right)}^{4}+d_{\left(a^{*}, b^{*}\right)}-d_{(a, b)}=2^{n+1}+1-2^{b}-\frac{a}{n} .
\end{aligned}
$$

Attaching $x_{i, j}^{1}$ and $x_{i, j}^{3}$ to $G_{i, j}$ is slightly more elaborate. We want to ensure that for any two pairs $(a, b),\left(a, b^{\prime}\right) \in S_{i, j}$ that agree on the first component, we have $d_{(a, b)}^{1}=d_{\left(a, b^{\prime}\right)}^{1}$. For that purpose, we add a path $U_{i, j}^{1}=u_{1}^{1}, \ldots, u_{n}^{1}$ and set the length of every edge $\left\{u_{\lambda}^{1}, u_{\lambda+1}^{1}\right\}$ to $2^{\lambda}$. Moreover, we add the edge $\left\{u_{n}^{1}, x_{i, j}^{1}\right\}$ of length $2^{n}$. For every $b \in[n]$, consider the vertex $v_{\left(a^{*}, b\right)}^{1}$ that is furthest from $z_{i, j}^{1} \cdot{ }^{3}$ We call it also the $b$-portal $\rho_{b}^{1}$. We attach it to $u_{b}^{1}$ through an edge of length $2^{b}-a^{*} / n$, the so called $b$-portal edge. It follows that for $(a, b) \in S_{i, j}$ we have

$$
\operatorname{dist}\left(v_{(a, b)}^{1}, u_{b}^{1}\right)=2^{b}-\frac{a^{*}}{n}+d_{\left(a^{*}, b\right)}-d_{(a, b)}=2^{b}-\frac{a}{n}
$$

and

$$
\operatorname{dist}\left(u_{b}^{1}, x_{i, j}^{1}\right)=\sum_{\lambda=b}^{n} 2^{\lambda}=2^{n+1}-2^{b},
$$

and hence we have

$$
d_{(a, b)}^{1}=2^{n+1}-\frac{a}{n} .
$$

Similarly we proceed with the vertices contained in $O_{i, j}^{3}$. We add a path $U_{i, j}^{3}=$ $u_{1}^{3}, \ldots, u_{n}^{3}$, set the length of every edge $\left\{u_{\lambda}^{3}, u_{\lambda+1}^{3}\right\}$ to $2^{\lambda}$ and add the edge $\left\{u_{n}^{3}, x_{i, j}^{3}\right\}$

\footnotetext{
2 Here the minimum is taken w.r.t. the lexical order as defined previously.

3 This means $a^{*}=\max _{(a, b) \in S_{i, j}} a$.
} 
of length $2^{n}$. For $b \in[n]$ we use the vertex $v_{\left(a^{*}, b\right)}^{3}$ that is closest to $z_{i, j}^{3}$ as the $b$-portal $\rho_{b}^{3}$ and attach it to $u_{b}^{3}$ trough a portal edge of length $2^{b}-1+a^{*} / n$. It follows that

$$
d_{(a, b)}^{3}=2^{n+1}-1+\frac{a}{n}
$$

To complete the construction, we connect the individual gadgets in a grid-like fashion. For $i \in[n-1]$ we connect $x_{i, j}^{3}$ and $x_{i+1, j}^{1}$ through a path $P_{i, j}$ of length 1 that consists of $(n+1)$ edges of length $1 /(n+1)$ each. Moreover, for $j \in[n-1]$ we connect $x_{i, j}^{2}$ and $x_{i, j+1}^{4}$ through a path $P_{i, j}^{\prime}=w_{1}, \ldots, w_{n}$ where $w_{1}=x_{i, j+1}^{4}$ and $w_{n}=x_{i, j}^{4}$. We set the length of every edge $\left\{w_{\lambda+1}, w_{\lambda}\right\}$ to $2^{\lambda}$ which implies that $\ell\left(P_{i, j}^{\prime}\right)=2^{n}-2$. The resulting graph $G_{\mathcal{I}}$ can be constructed in polynomial time from the given $\mathrm{GT}_{\leq}$-instance $\mathcal{I}$.

\subsection{Graph properties}

We now show some basic properties of $G_{\mathcal{I}}$ that will be useful to prove the correctness of our reduction and to obtain bounds on several graph parameters. We first observe that all shortest paths between the cycle $O_{i, j}$ and a path $U_{i, j}^{h}$ have a certain structure (cf. Fig. 2).

Lemma 1 Let $a, b, b^{\prime} \in[n]$ and $h \in\{1,3\}$. For $\beta \in[n]$ denote the path $\pi\left(v_{(a, b)}^{h}, \rho_{\beta}^{h}\right) \circ\left\{\rho_{\beta}^{h}, u_{\beta}^{h}\right\} \circ \pi\left(u_{\beta}^{h}, u_{b^{\prime}}^{h}\right)$ by $P_{\beta}$.

(a) If $b^{\prime} \geq b$, the shortest path from $v_{(a, b)}^{h}$ to $u_{b^{\prime}}^{h}$ is $P_{b}$.

(b) If $b^{\prime}<b$, the shortest path from $v_{(a, b)}^{h}$ to $u_{b^{\prime}}^{h}$ is $P_{b^{\prime}}$.

Proof Any shortest path from $v_{(a, b)}^{h}$ to $u_{b^{\prime}}^{h}$ needs to contain some portal edge $\left\{\rho_{\beta}^{h}, u_{\beta}^{h}\right\}$. We only prove case (a) for $h=1$, the remaining cases can be shown similarly.

Let $\beta \in[n]$ and let $\rho_{\beta}^{1}=v_{(\alpha, \beta)}^{1}$ be the $\beta$-portal. The path $P_{\beta}$ has length $\operatorname{dist}\left(v_{(a, b)}^{1}, \rho_{\beta}^{1}\right)+\operatorname{dist}\left(\rho_{\beta}^{1}, u_{\beta}^{1}\right)+\operatorname{dist}\left(u_{\beta}^{1}, u_{b^{\prime}}^{1}\right)$, which equals

$$
\left|2^{\beta}-2^{b}+\frac{(\alpha-a)}{n}\right|+2^{\beta}-\frac{\alpha}{n}+\left|2^{b^{\prime}}-2^{\beta}\right| .
$$

This means that $\ell\left(P_{b}\right)=2^{b^{\prime}}-a / n$, and

$$
\ell\left(P_{\beta}\right)= \begin{cases}2^{b^{\prime}}+2^{b}-2^{\beta}+\frac{a-2 \alpha}{n} & \text { for } \beta<b \\ 2^{b^{\prime}}+2^{\beta}-2^{b}-\frac{\alpha}{n} & \text { for } b<\beta \leq b^{\prime} \\ 3 \cdot 2^{\beta}-2^{b}-2^{b^{\prime}}-\frac{a}{n} & \text { for } \beta \geq b^{\prime} .\end{cases}
$$

It follows that for $\beta \neq b$ we have $\ell\left(P_{\beta}\right)>\ell\left(P_{b}\right)$, and hence, $P_{b}$ is the shortest path from $v_{(a, b)}^{h}$ to $u_{b^{\prime}}^{h}$. 


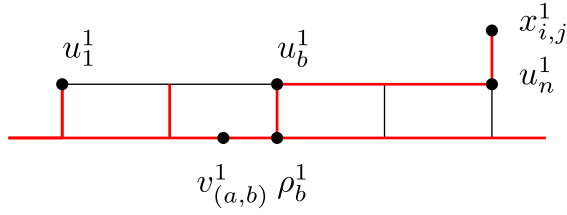

(a) The shortest path tree of a vertex $v_{(a, b)}^{1}$.

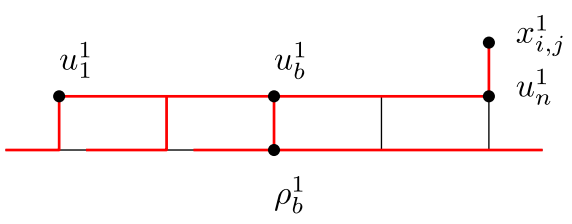

(b) The shortest path tree of a vertex $u_{b}^{1}$.

Fig. 2 Illustration of the shortest path structure as shown in Lemma 1. Shortest paths are drawn using thick red lines

Moreover, it holds that for any vertex $v$ of the graph $G_{\mathcal{I}}$, there is some central vertex $y_{i, j}$ not too far away.

Lemma 2 For every vertex $v$, we have $\min _{(i, j)} \operatorname{dist}\left(v, y_{i, j}\right) \leq 2^{n+2}+2^{n+1}$.

Proof Assume first that $v$ is contained in some gadget $G_{i, j}$. If $v$ is contained in the cycle $O_{i, j}$, the distance to the closest vertex $z_{i, j}^{h}$ is at most $2^{n+1}$ as every edge length is a multiple of $1 / n$ and the subpath $O_{i, j}^{h}$ between $z_{i, j}^{h}$ and $z_{i, j}^{h \boxplus 1}$ has length $2^{n+2}+1 / n$. Moreover, we have $\operatorname{dist}\left(z_{i, j}^{h}, y_{i, j}\right)=2^{n+1}+1$, and hence, the distance between any $v \in O_{i, j}$ and $y_{i, j}$ is bounded by $2^{n+2}+1$.

Consider now some vertex $x_{i, j}^{h}$. The distance from $x_{i, j}^{h}$ to any vertex $v_{(a, b)}^{h}$ is $d_{(a, b)}^{h}$ and the is a path from $d_{(a, b)}^{h}$ to $y_{i, j}$ via $z_{i, j}^{h}$ of length $d_{(a, b)}+2^{n+1}+1$. It follows, that

$$
\operatorname{dist}\left(x_{i, j}^{h}, y_{i, j}\right) \leq d_{(a, b)}^{h}+d_{(a, b)}+2^{n+1}+1 \leq 2^{n+2}+2^{n}+2,
$$

where the last inequality follows from the fact that $d_{(a, b)}^{h}+d_{(a, b)} \leq 2^{n+1}+2^{n}+1$, which is easy to verify. Assume now that $v \in u_{b}^{h}$ for some $h \in\{1,3\}$ and $b \in[n]$. The shortest path from $v$ to $y_{i, j}$ passes through the portal edge $\left\{u_{b}^{h}, \rho_{b}^{h}\right\}$, which has length at most $2^{b}$, and the vertex $z_{i, j}^{h}$. The distance from $\rho_{b}^{h}$ to $z_{i, j}^{h}$ is at most $2^{b}$ and it follows that

$$
\operatorname{dist}\left(u_{b}^{h}, y_{i, j}\right) \leq 2^{b}+2^{b}+2^{n+1}+1 \leq 2^{n+2}+1
$$

It remains to consider the case where $v$ is not contained in any gadget. If this holds, $v$ is contained in some path $P_{i, j}$ or $P_{i, j}^{\prime}$ between two gadgets. The lengths of these paths is bounded by $2^{n}-2$ and hence, there is some vertex $x_{i, j}^{h} \operatorname{such}$ that $\operatorname{dist}\left(v, x_{i, j}^{h}\right) \leq 2^{n}-2$. It follows from Equation 1, that $\operatorname{dist}\left(v, y_{i, j}\right) \leq 2^{n+2}+2^{n+1}$.

\subsection{Correctness of the reduction}

We show now that the $\mathrm{GT}_{\leq}$-instance $\mathcal{I}$ has a solution if and only if the $k$-CENTER instance $G_{\mathcal{I}}$ has a solution of cost at most $2^{n+1}$ for $k=5 \chi^{2}$ centers.

Lemma $3 A$ solution for the $\mathrm{GT}_{\leq}$-instance $\mathcal{I}$ implies a solution for the $k$-CENTER instance $G_{\mathcal{I}}$ of cost at most $2^{n+1}$. 
Proof For $(i, j) \in[n]^{2}$ let $s_{i, j}$ be the pair from $S_{i, j}$ that is chosen in a solution of $\mathcal{I}$. For the $k$-CENTER instance $G_{\mathcal{I}}$, we choose a center set $C$ of size $5 \chi^{2}$ by selecting from every gadget $G_{i, j}$ the central vertex $y_{i, j}$ and the four vertices $v_{s_{i, j}}^{1}, \ldots, v_{s_{i, j}}^{4}$. We show that $C$ has cost at most $2^{n+1}$.

Consider a gadget $G_{i, j}$ and the four chosen centers $v_{(a, b)}^{1}, \ldots, v_{(a, b)}^{4}$. It holds that the distance between any two neighboring centers $v_{(a, b)}^{h}$ and $v_{(a, b)}^{h \boxplus 1}$ is $2^{n+2}+1 / n$ and moreover, the length of every edge of the cycle $O_{i, j}$ is a multiple of $1 / n$. Hence, it follows that for every vertex $v \in O_{i, j}$ there is some center vertex $v_{(a, b)}^{h}$ at distance at most $2^{n+1}$. Consider some vertex $u_{b^{\prime}}^{h}$ for $h \in\{1,3\}$. It follows from Lemma 1 , that $\operatorname{dist}\left(v_{(a, b)}^{h}, u_{b^{\prime}}^{h}\right) \leq 2^{n+1}$. Finally, the vertex $y_{i, j}$ is chosen as a center. This means that the complete gadget $G_{i, j}$ is contained in the five balls of radius $2^{n+1}$ around $y_{i, j}$ and $v_{(a, b)}^{1}, \ldots, v_{(a, b)}^{4}$.

It remains to show that the chosen centers cover all paths $P_{i, j}$ and $P_{i, j}^{\prime}$ that connect the individual gadgets. Consider two neighboring gadgets $G_{i, j}$ and $G_{i+1, j}$ and let $s_{i, j}=(a, b)$ and $s_{i+1, j}=\left(a^{\prime}, b^{\prime}\right)$ be the corresponding pairs from the solution of $\mathcal{I}$. We have $a \leq a^{\prime}$. From $G_{i, j}$ we have chosen a center $v_{(a, b)}^{3}$ that has distance $d_{(a, b)}^{3}$ to $x_{i, j}^{3}$. Similarly, we have chosen some $v_{\left(a^{\prime}, b^{\prime}\right)}^{1}$ from $G_{i+1, j}$ whose distance to $x_{i+1, j}^{1}$ is $d_{\left(a^{\prime}, b^{\prime}\right)}^{1}$. The path $P_{i, j}$ between $x_{i, j}^{3}$ and $x_{i+1, j}^{1}$ has length 1 , and hence the distance between the two considered centers is

$d_{(a, b)}^{3}+\ell\left(P_{i, j}\right)+d_{\left(a^{\prime}, b^{\prime}\right)}^{1}=2^{n+1}-1+\frac{a}{n}+1+2^{n+1}-\frac{a^{\prime}}{n}=2^{n+2}+\frac{a-a^{\prime}}{n} \leq 2^{n+2}$.

This means that $P_{i, j}$ can be covered with balls of radius $2^{n+1}$ around $v_{\left(a^{\prime}, b^{\prime}\right)}^{1}$ and $v_{(a, b)}^{3}$. Similarly, $b \leq b^{\prime}$ yields

$$
\begin{aligned}
d_{(a, b)}^{2}+\ell\left(P_{i, j}^{\prime}\right)+d_{\left(a^{\prime}, b^{\prime}\right)}^{4} & =2^{n}+2^{b}+\frac{a}{n}+2^{n}-2+2^{n+1}+1-2^{b^{\prime}}-\frac{a^{\prime}}{n} \\
& =2^{n+2}-1+2^{b}-2^{b^{\prime}}+\frac{a-a^{\prime}}{n}<2^{n+2}
\end{aligned}
$$

Hence, any vertex contained in a path $P_{i, j}^{\prime}$ has distance at most $2^{n+1}$ from a chosen center.

In the next lemma we show that every solution for $G_{\mathcal{I}}$ of cost at most $2^{n+1}$ contains four equidistant vertices $v_{(a, b)}^{1}, \ldots, v_{(a, b)}^{4}$ from every $G_{i, j}$, which yield a solution for $\mathcal{I}$. This completes our correctness proof.

Lemma 4 A solution for the $k$ - CENTER instance $G_{\mathcal{I}}$ of cost at most $2^{n+1}$ implies a solution for the $\mathrm{GT}_{\leq}$-instance $\mathcal{I}$.

Proof Let $C$ be a solution for $G_{\mathcal{I}}$ of cost at most $2^{n+1}$. Consider a gadget $G_{i, j}$. The central vertex $y_{i, j}$ has distance at least $2^{n+1}+1$ to any other vertex. Hence we have $y_{i, j} \in C$. Let $C_{i, j}$ be the remaining centers from $C$ that have distance at most $2^{n+1}$ from any vertex of $G_{i, j}$. As $k=5 \chi^{2}$, there are at most $4 \chi^{2}$ such centers in total. We first show that every $C_{i, j}$ consists of exactly 4 vertices contained in the cycle $O_{i, j}$. 
Claim For $(i, j) \in[n]^{2}$ we have $C_{i, j} \subseteq O_{i, j}$ and $\left|C_{i, j}\right|=4$.

To prove this, let $(A, B)=\max S_{i, j}$ and let $h \in[4]$. We show that $\psi_{(A, B)}^{h}$ can only be covered through vertices from $G_{i, j} \backslash\left\{x_{i, j}^{1}, \ldots, x_{i, j}^{4}\right\}$.

Consider some vertex $x_{i, j}^{h^{\prime}}$. The shortest path from $x_{i, j}^{h^{\prime}}$ to $\psi_{(A, B)}^{h}$ has to pass trough either $v_{(A, B)}^{h}$ or $z_{i, j}^{h}$. The distance from $\psi_{(A, B)}^{h}$ to $v_{(A, B)}^{h}$ is $2^{n+1}$ whereas the distance from $\psi_{(A, B)}^{h}$ to $z_{i, j}^{h}$ is $2^{n+1}+1 / n-2^{B}+1-A / n>2^{n}$. Moreover, the distance from $x_{i, j}^{h^{\prime}}$ to any vertex in the cycle $O_{i, j}$ is at least $2^{n}$. It follows that $\operatorname{dist}\left(x_{i, j}^{h^{\prime}}, \psi_{(A, B)}^{h}\right)>2^{n+1}$ and hence, $\psi_{(A, B)}^{h}$ cannot be covered through $x_{i, j}^{h^{\prime}}$ or any vertex not contained in the gadget $G_{i, j}$.

Moreover, any two of the vertices $\psi_{(A, B)}^{1}, \ldots, \psi_{(A, B)}^{4}$ have distance at least $2^{n+2}+$ $1 / n$ and hence we need at least 4 centers to cover them with balls of radius $2^{n+1}$. This implies that $C_{i, j} \subseteq G_{i, j} \backslash\left\{x_{i, j}^{1}, \ldots, x_{i, j}^{4}\right\}$ and $\left|C_{i, j}\right|=4$.

Assume now that $C_{i, j} \nsubseteq O_{i, j}$, which means that some vertex $u_{b}^{h} \in C_{i, j}$ was chosen as a center. Let $v_{(a, b)}^{h}$ be the corresponding $b$-portal. Lemma 1 implies that the distance from $u_{b}^{h}$ to any of the vertices $\psi_{(a, b)}^{1}, \ldots, \psi_{(a, b)}^{4}$ is more than $2^{n+1}$. Moreover, the pairwise distance of $\psi_{(a, b)}^{1}, \ldots, \psi_{(a, b)}^{4}$ is at least $2^{n+2}+1 / n$. This means that apart from $u_{b}^{h}$, the set $C_{i, j}$ needs to contain 4 more centers, which contradicts $\left|C_{i, j}\right|=4$. Hence we obtain $C_{i, j} \subseteq O_{i, j}$.

We now show, that every $C_{i, j}$ contains four equidistant centers $v_{(a, b)}^{h}$, i.e., any two consecutive centers on $O_{i, j}$ are at distance $2^{n+2}+1 / n$ from each other.

Claim For $(i, j) \in[n]^{2}$ we have $C_{i, j}=\left\{v_{(a, b)}^{1}, \ldots, v_{(a, b)}^{4}\right\}$ for $(a, b) \in S_{i, j}$.

Let $(\alpha, \beta)$ be the minimum of $S_{i, j}$. Consider the vertex $x_{i, j}^{1}$. Its distance to $z_{i, j}^{1}, \psi_{(\alpha, \beta)}^{1}$ and any vertex of $O_{i-1, j}$ is more than $2^{n+1}$. Hence, it must be covered through some vertex $v_{(a, b)}^{1}$ where $(a, b) \in S_{i, j}$. Consider the vertices $\psi_{(a, b)}^{\prime 1}, \psi_{(a, b)}^{2}, \psi_{(a, b)}^{\prime 2}, \psi_{(a, b)}^{3}, \psi_{(a, b)}^{\prime 3}, \psi_{(a, b)}^{4}$. None of them is contained in the ball of radius $2^{n+1}$ around $v_{(a, b)}^{1}$. Moreover, for $h \in\{1,2,3\}$, the distance between $\psi_{(a, b)}^{\prime h}$ and $\psi_{(a, b)}^{h \boxplus 1}$ is $2^{n+2}$, whereas the distance between $\psi_{(a, b)}^{\prime 1}$ and $\psi_{(a, b)}^{\prime 2}$ and the distance between $\psi_{(a, b)}^{3}$ and $\psi_{(a, b)}^{4}$ are both $2^{n+2}+1 / n$. This means that the whole cycle $O_{i, j}$ can only be covered with 4 balls of radius $2^{n+1}$ if we have $\left\{v_{(a, b)}^{2}, v_{(a, b)}^{3}, v_{(a, b)}^{4}\right\} \subseteq C_{i, j}$.

Finally we show that the sets $C_{i, j}$ yield a solution for $\mathcal{I}$.

Claim For $(i, j) \in[n]^{2}$ choosing $s_{i, j}=(a, b)$ where $v_{(a, b)}^{1} \in C_{i, j}$ yields a solution for the $\mathrm{GT}_{\leq}$-instance $\mathcal{I}$.

Let $s_{i, j}=(a, b)$ and $s_{i+1, j}=\left(a^{\prime}, b^{\prime}\right)$ and assume that $a>a^{\prime}$. Consider the path $P_{i, j}$ connecting the vertices $x_{i, j}^{3}$ and $x_{i+1, j}^{1}$. As the path $P_{i, j}$ consists of $n+1$ edges of length $1 /(n+1)$, it contains a vertex $w$ that has distance $1-a /(n+1)$ from $x_{i, j}^{3}$ and distance $a /(n+1)$ from $x_{i+1, j}^{1}$. It follows that the distances from $w$ to the closest 
centers in $G_{i, j}$ and $G_{i+1, j}$ are

$$
\begin{aligned}
& d_{(a, b)}^{3}+1-\frac{a}{n+1}=2^{n+1}-1+\frac{a}{n}+1-\frac{a}{n+1}>2^{n+1} \text { and } \\
& d_{\left(a^{\prime}, b^{\prime}\right)}^{1}+\frac{a}{n+1}=2^{n+1}-\frac{a^{\prime}}{n}+\frac{a}{n+1}>2^{n+1},
\end{aligned}
$$

respectively. This contradicts the fact that $C$ is a solution for the $k$-CENTER instance, and hence $a \leq a^{\prime}$. Similarly, let $s_{i, j}=(a, b)$ and $s_{i, j+1}=\left(a^{\prime}, b^{\prime}\right)$ and assume that $b>b^{\prime}$. Consider the path $P_{i, j}^{\prime}=w_{1} \ldots w_{n}$ connecting $x_{i, j}^{2}$ and $x_{i, j+1}^{4}$. Recall that every edge $\left\{w_{\lambda+1}, w_{\lambda}\right\}$ has length $2^{\lambda}$ and hence we have $\operatorname{dist}\left(x_{i, j}^{2}, w_{b}\right)=2^{n}-2^{b}$ and $\operatorname{dist}\left(w_{b}, x_{i, j+1}^{4}\right)=2^{b}-2$. It follows that the distances from $w_{b}$ to the closest centers in $G_{i, j}$ and $G_{i, j+1}$ are

$$
\begin{aligned}
& d_{(a, b)}^{2}+2^{n}-2^{b}=2^{n}+2^{b}+\frac{a}{n}+2^{n}-2^{b}=2^{n+1}+\frac{a}{n}>2^{n+1} \text { and } \\
& d_{\left(a^{\prime}, b^{\prime}\right)}^{4}+2^{b}-2=2^{n+1}+1-2^{b^{\prime}}+\frac{a^{\prime}}{n}+2^{b}-2 \geq 2^{n+1}+\frac{a^{\prime}}{n}>2^{n+1},
\end{aligned}
$$

respectively, which gives a contradiction. It follows that $b \leq b^{\prime}$ and hence, choosing $s_{i, j}=(a, b)$ for $v_{(a, b)}^{1} \in C_{i, j}$ yields a solution for $\mathcal{I}$.

This completes the proof as any solution $C$ of cost at most $2^{n+1}$ for the $k$-CENTER instance $G_{\mathcal{I}}$ implies a solution for the $\mathrm{GT}_{\leq}$-instance $\mathcal{I}$.

\section{Bounds on graph parameters}

In this part we show bounds on the doubling dimension, the highway dimension, the skeleton dimension and the pathwidth of the graph $G_{\mathcal{I}}$, which imply Theorem 1 . To bound the doubling dimension, we exploit the fact that the individual gadgets $G_{i, j}$ are connected in a grid-like fashion. This means that we can bound the diameter of balls within this grid. For that purpose, let $A_{i, j}(d)=\left\{\left(i^{\prime}, j^{\prime}\right) \in[\chi]^{2}|| i^{\prime}-i|+| j^{\prime}-j \mid \leq d\right\}$. Moreover, let $V_{i, j}(d)$ be the vertices of all gadgets $G_{i^{\prime}, j^{\prime}}$ satisfying $\left(i^{\prime}, j^{\prime}\right) \in A_{i, j}(d)$ and the vertices on the paths $P_{i^{\prime}, j^{\prime}}$ and $P_{i^{\prime}, j^{\prime}}^{\prime}$ between these gadgets. We now bound the diameter of the graph induced by $V_{i, j}(d)$.

Lemma 5 Consider the graph induced by $V_{i, j}(d)$. Its diameter is at most $\left(2^{n+3}+\right.$ $\left.2^{n+1}+2^{n}+2\right) \cdot(2 d+1)$. Moreover, if $\left|A_{i, j}(d)\right|=(2 d+1)^{2}$, i.e. $A_{i, j}(d)$ contains all possible index pairs, the diameter is at least $\left(2^{n+2}+2^{n}\right) \cdot(2 d+1)$.

Proof Let $(i, j) \in[\chi]$. We first bound the distance between any $x_{i, j}^{h}$ and $x_{i, j}^{h^{\prime}}$. In particular we show that for any $h, h^{\prime} \in[4]$ where $h \neq h^{\prime}$ we have

$$
2^{n+2}+2^{n}<\operatorname{dist}\left(x_{i, j}^{h}, x_{i, j}^{h^{\prime}}\right) \leq 2^{n+3}+2^{n+1}+4 .
$$

The upper bound follows directly from Equation 1 in the proof of Lemma 2. For the lower bound, observe that the shortest path between $x_{i, j}^{h}$ and $x_{i, j}^{h^{\prime}}$ needs to pass through 
two vertices $v_{(a, b)}^{h}$ and $v_{\left(a^{\prime}, b^{\prime}\right)}^{h^{\prime}}$ of the cycle $O_{i, j}$. It holds that the distance from $x_{i, j}^{h}$ to $v_{(a, b)}^{h}$ and from $x_{i, j}^{h^{\prime}}$ to $v_{\left(a^{\prime}, b^{\prime}\right)}^{h^{\prime}}$ are $d_{(a, b)}^{h}$ and $d_{\left(a^{\prime}, b^{\prime}\right)}^{h^{\prime}}$, which are both at least $2^{n}$. Moreover, the distance from $v_{(a, b)}^{h}$ to $v_{\left(a^{\prime}, b^{\prime}\right)}^{h^{\prime}}$ is minimized, if $v_{(a, b)}^{h}=v_{(n, n)}^{h}$ and $v_{\left(a^{\prime}, b^{\prime}\right)}^{h^{\prime}}=v_{(1,1)}^{h \boxplus 1}$. As $\operatorname{dist}\left(v_{(n, n)}^{h}, v_{(1,1)}^{h \boxplus 1}\right)=2^{n+2}+1 / n+d_{(1,1)}-d_{(n, n)}=2^{n+1}+2^{n}+1+1 / n$, a lower bound of $2 \cdot 2^{n}+2^{n+1}+2^{n}+1+1 / n>2^{n+2}+2^{n}$ on $\operatorname{dist}\left(x_{i, j}^{h}, x_{i, j}^{h^{\prime}}\right)$ follows.

Consider now the graph induced by $V_{i, j}(d)$. Any shortest path in this graph traverses at most $2 d+1$ gadgets and contains at most $2 d$ paths between two gadgets. These paths have length at most $2^{n}-2$. Moreover, it follows from the proof of Lemma 2 that the diameter of a single gadget is at most $2^{n+3}+2^{n+1}+4$. This means that the distance of any shortest path is upper bounded by $(2 d+1) \cdot\left(2^{n+3}+2^{n+1}+2^{n}+2\right)$.

If $\left|A_{i, j}(d)\right|=(2 d+1)^{2}$, the shortest path from $x_{i-d, j}^{1}$ to $x_{i+d, j}^{4}$ has to traverse $2 d+1$ gadgets hence a lower bound of $(2 d+1) \cdot\left(2^{n+2}+2^{n}\right)$ on the diameter of the graph induced by $V_{i, j}(d)$ follows.

This allows us to show that the doubling dimension of $G_{\mathcal{I}}$ is constant.

\section{Lemma 6 The graph $G_{\mathcal{I}}$ is planar and has constant doubling dimension.}

Proof It can be seen easily that $G_{\mathcal{I}}$ is planar. Recall that a graph has doubling dimension at most $\lambda$ if any ball of radius $2 r$ can be covered with $2^{\lambda}$ balls of radius $r$.

To bound the doubling dimension of $G_{\mathcal{I}}$, consider a ball $B_{2 r}(v)$ of radius $2 r$ around some vertex $v \in V$. Lemma 2 implies that there is a vertex $y_{i, j}$ satisfying $\operatorname{dist}\left(v, y_{i, j}\right) \leq 2^{n+2}+2^{n+1}$. It follows that the ball $B_{2 r}(v)$ is contained in the ball around $y_{i, j}$ that has radius $2^{n+2}+2^{n+1}+2 r$. Moreover, Lemma 5 implies that the latter ball in turn is contained in $V_{i, j}(d)$ if $2 \cdot\left(2^{n+2}+2^{n+1}+2 r\right) \leq\left(2^{n+2}+2^{n}\right) \cdot(2 d+1)$. This is true for $2 d+1=6 r / 2^{n+2}$ and $r \geq 2^{n+2}+2^{n+1}$.

We now show that we can cover the vertices $V_{i, j}(d)$ through a constant number of balls that have radius $r$ and are centered at vertices $y_{i^{\prime}, j^{\prime}}$. Lemma 5 implies that for every $\left(i^{\prime}, j^{\prime}\right) \in[\chi]^{2}$, the ball $B_{r}\left(y_{i^{\prime}}, j^{\prime}\right)$ contains the set $V_{i^{\prime}, j^{\prime}}\left(d^{\prime}\right)$ if $2 r \geq\left(2^{n+3}+\right.$ $\left.2^{n+1}+2^{n}+2\right) \cdot\left(2 d^{\prime}+1\right)$. This is the case for $2 d^{\prime}+1=2 r / 2^{n+4}$. As we want $V_{i^{\prime}, j^{\prime}}\left(d^{\prime}\right)$ to be nonempty, we require $d^{\prime} \geq 0$, which holds for $r \geq 2^{n+3}$. Hence it suffices to show that there is a constant number of sets $V_{i^{\prime}, j^{\prime}}\left(d^{\prime}\right)$ whose union contains $V_{i, j}(d)$.

As it was observed in (Feldmann and Marx 2020), the index set $A_{i, j}(d)$ is contained in the union of $\left\lceil\frac{2 d+1}{2 d^{\prime}+1}\right\rceil^{2}$ index sets $A_{i^{\prime}, j^{\prime}}\left(d^{\prime}\right)$. It follows that we can cover the vertices $V_{i, j}(d)$ through $\left\lceil\frac{2 d+1}{2 d^{\prime}+1}\right\rceil^{2}$ vertex sets $V_{i^{\prime}, j^{\prime}}\left(d^{\prime}\right)$. Hence, for $r \geq 2^{n+3}$ we can cover $B_{2 r}(v)$ with $\left\lceil\frac{2 d+1}{2 d^{\prime}+1}\right\rceil^{2}=\left\lceil\frac{6 r / 2^{n+2}}{2 r / 2^{n+4}}\right\rceil^{2}=144$ balls of radius $r$.

Assume now that $r<2^{n+3}$. We already showed that $B_{2 r}(v)$ is contained in $V_{i, j}(d)$ if $2 d+1=6 r / 2^{n+2}<12$, which implies $d<6$. Hence, the ball $B_{2 r}(v)$ intersects at most $\left|A_{i, j}(5)\right| \leq(2 \cdot 5+1)^{2}=121$ gadgets $G_{i^{\prime}, j^{\prime}}$. We show that we can cover any of these gadgets $G_{i^{\prime}, j^{\prime}}$ and the paths to its neighboring gadgets through a constant number of ball $B_{r}(w)$.

If $r \geq 2^{n+1}$, we can choose the 9 balls centered at $y_{i^{\prime}, j^{\prime}}, z_{i^{\prime}, j^{\prime}}^{h}$ and $x_{i^{\prime}, j^{\prime}}^{h}$ where $h \in[4]$, as for every $w \in O_{i^{\prime}, j^{\prime}}$ there is some $z_{i^{\prime}, j^{\prime}}^{h} \operatorname{satisfying} \operatorname{dist}\left(z_{i^{\prime}, j^{\prime}}^{h}, w\right) \leq 2^{n+1}$, for $h \in\{1,3\}$ and $b \in[n]$ it holds that $\operatorname{dist}\left(x_{i^{\prime}, j^{\prime}}^{h}, u_{b}^{h}\right) \leq 2^{n+1}$ and the length the paths to the neighboring gadgets have length at most $2^{n}-2$. 
Let now $r<2^{n+1}$. If $v=y_{i^{\prime}, j}$, i.e. the ball $B_{2 r}(v)$ is centered at $y_{i^{\prime}, j}$, we can choose $X_{i^{\prime}, j^{\prime}}=\left\{y_{i^{\prime}, j^{\prime}}, z_{i^{\prime}, j^{\prime}}^{h} \mid h \in[4]\right\}$. Otherwise, $B_{2 r}(v) \cap O_{i^{\prime}, j^{\prime}}$ is a subpath of $O_{i^{\prime}, j^{\prime}}$ that has length at most $4 r$, which can be covered by 4 balls of radius $r$. Similarly, we can also cover $B_{2 r}(v) \cap U_{i^{\prime}, j^{\prime}}^{h}, B_{2 r}(v) \cap P_{i^{\prime}, j^{\prime}}$ and $B_{2 r}(v) \cap P_{i^{\prime}, j^{\prime}}^{\prime}$ with 4 balls of radius $r$ each. This means that we can cover $B_{2 r} \cap G_{i^{\prime}, j^{\prime}}$ through a constant number of balls of radius $r$.

It follows that we can cover any ball $B_{2 r}(v)$ for any $v \in V$ and any $r>0$ with a constant number of balls of radius $r$, which completes the proof.

We next bound the highway dimension of $G_{\mathcal{I}}$.

Lemma 7 The graph $G_{\mathcal{I}}$ has highway dimension $h d \in \mathcal{O}\left(\chi^{2}\right)$.

Proof For any radius $r>0$ we specify a set $H_{r}$ such that every shortest path $\pi$ satisfying $\ell(\pi)>r$ intersects $H_{r}$ and moreover, for every vertex $v \in V$ we have $\left|H_{r} \cap B_{4 r}(v)\right| \in \mathcal{O}\left(\chi^{2}\right)$. Let

$$
X=\left\{y_{i, j}, x_{i, j}^{h}, z_{i, j}^{h} \mid(i, j) \in[\chi]^{2}, h \in[4]\right\} .
$$

For $r \geq 2^{n+2}$ we choose $H_{r}=X$. We have $\left|H_{r}\right|=9 \chi^{2}$ and hence for every vertex $v \in V$ we have $\left|H_{r} \cap B_{4 r}(v)\right| \in \mathcal{O}\left(\chi^{2}\right)$. We show now that any shortest path of length more than $r$ intersects $H_{r}$. Clearly, all shortest paths that are not completely contained within one single gadget are hit by $H_{r}$ as all $x_{i, j}^{h}$ are contained in $H_{r}$ and the paths $P_{i, j}$ and $P_{i, j}^{\prime}$ between the individual gadgets have length at most $2^{n}-2$. Consider some gadget $G_{i, j}$. All edges of the cycle $O_{i, j}$ have length at least $1 / n$ and for any $h \in$ [4] we have $\operatorname{dist}\left(z_{i, j}^{h}, z_{i, j}^{h \boxplus 1}\right)=2^{n+2}+1 / n$. Hence, any subpath of $O_{i, j}$ that has length at least $2^{n+2}$ intersects $H_{r}$. Moreover, for $h \in\{1,3\}$, the path $U_{i, j}^{h}$ has length $2^{n}-2$.

It remains to consider some shortest path $\pi(s, t)$ where $s \in O_{i, j}$ and $t \in U_{i, j}^{h}$. Let $t=u_{b}^{h}$. According to Lemma 1 , the shortest path $\pi(s, t)$ traverses exactly one portal edge $\left\{\rho_{\beta}^{h}, u_{\beta}^{h}\right\}$ where $\beta \in[b]$. This means that $\operatorname{dist}(s, t)=\operatorname{dist}\left(s, \rho_{\beta}^{h}\right)+\operatorname{dist}\left(\rho_{\beta}^{h}, u_{b}^{h}\right) \leq$ $\operatorname{dist}\left(s, \rho_{\beta}^{h}\right)+2^{b}$. The vertex $s$ is contained in the shortest path $\pi\left(z_{i, j}^{h}, \rho_{\beta}^{h}\right)$ or in $\pi\left(\rho_{\beta}^{h}, z_{i, j}^{h \boxplus 1}\right)$. In the first case we have $\operatorname{dist}\left(s, \rho_{\beta}^{h}\right)<\operatorname{dist}\left(z_{i, j}^{h}, \rho_{\beta}^{h}\right) \leq 2^{\beta}$. This implies that $\operatorname{dist}(s, t)<2^{\beta}+2^{b} \leq 2^{n+1}$. In the second case we have $\operatorname{dist}\left(s, \rho_{\beta}^{h}\right) \leq 2^{n+2}-2^{\beta}$ and moreover Lemma 1 implies that $\beta=b$. Hence we obtain $\operatorname{dist}(s, t) \leq 2^{n+2}-$ $2^{\beta}+2^{\beta}=2^{n+2}$. This means that every shortest path of length more than $r \geq 2^{n+2}$ is hit by $H_{r}$.

Let now $r<2^{n+2}$. For a shortest path $p=v_{1}, \ldots, v_{v}$ and $q>0$ let $p^{\langle q\rangle}$ be a $q$-cover of $p$, i.e., we have $p^{\langle q\rangle} \subseteq\left\{v_{1}, \ldots, v_{\nu}\right\}$ such that any subpath of $p$ that has length at least $q$ contains some node from $p^{\langle q\rangle}$. We consider $q$-covers $p^{\langle q\rangle}$ that are constructed greedily, i.e., we start with $p^{\langle q\rangle}=\left\{v_{1}\right\}$ and iteratively add the closest vertex that has distance at least $q$. For $(i, j) \in[\chi]^{2}$ let

$$
X_{i, j}=\bigcup_{h \in[4]} O_{i, j}^{h}{ }^{\langle r / 4\rangle} \cup \bigcup_{h \in\{1,3\}} U_{i, j}^{h}{ }^{\langle r / 4\rangle} \cup\left\{u_{n}^{1}, u_{n}^{3}\right\} \cup P_{i, j}^{\langle r / 4\rangle} \cup P_{i, j}^{\prime}{ }^{\langle r / 4\rangle}
$$


and choose $H_{r}=X \cup \bigcup_{(i, j) \in[\chi]^{2}} X_{i, j}$. Consider some shortest path $\pi(s, t)$ that has length more than $r$. Clearly, $\pi(s, t)$ is hit by $H_{r}$ if it contains some node from $X$ or it is a subpath of some cycle $O_{i, j}$, some path $U_{i, j}^{h}$ or some path $P_{i, j}$ or $P_{i, j}^{\prime}$. It remains to be shown that $\pi(s, t)$ is also hit by $H_{r}$ if $s \in O_{i, j}$ and $t \in U_{i, j}^{h}$. Let $t=u_{b}^{h}$. Lemma 1 implies that $\pi(s, t)$ consists of a subpath $p$ of $O_{i, j}$, a portal edge $\left\{\rho_{\beta}^{h}, u_{\beta}^{h}\right\}$ and a subpath $p^{\prime}$ of $U_{i, j}^{h}$. Assume that $\pi(s, t)$ is not hit by $H_{r}$. By the choice of $X_{i, j}$ we have $\ell(p)<r / 4$ and $\ell\left(p^{\prime}\right) \mid<r / 4$. This means that $\operatorname{dist}\left(\rho_{\beta}^{h}, u_{\beta}^{h}\right)>r / 2$. By construction of the graph $G_{\mathcal{I}}$ we have $\operatorname{dist}\left(\rho_{\beta}^{h}, u_{\beta}^{h}\right) \leq 2^{\beta}$ and hence $2^{\beta}>r / 2$. As we have $u_{\beta}^{h} \notin X_{i, j}$, it holds that $\beta \notin\{1, n\}$ and moreover it follows from the choice of $U_{i, j}^{h}\langle r / 4\rangle$, that $\operatorname{dist}\left(u_{\beta-1}^{h}, u_{\beta}^{h}\right) \leq r / 4$. However, by construction of $G_{\mathcal{I}}$ we have dist $\left(u_{\beta-1}^{h}, u_{\beta}^{h}\right)=2^{\beta-1}$, which implies $2^{\beta} \leq r / 2$, a contradiction to $2^{\beta}>r / 2$. This means that every shortest path of length more than $r$ is hit by $H_{r}$.

Finally we have to show that for every vertex $v \in V$ we have $\left|H_{r} \cap B_{4 r}(v)\right| \in \mathcal{O}\left(\chi^{2}\right)$. As for the ${ }^{r / 4}$-cover of some shortest path $p$ we have $\left|B_{4 r}(v) \cap p^{\langle r / 4\rangle}\right| \in \mathcal{O}(1)$, it follows that for every $(i, j) \in[\chi]^{2}$ we have $\left|B_{4 r}(v) \cap X_{i, j}\right| \in \mathcal{O}(1)$. Moreover there are $\chi^{2}$ different sets $X_{i, j}$ and we have $|X|=9 \chi^{2}$, which implies $\left|H_{r} \cap B_{4 r}(v)\right| \in \mathcal{O}\left(\chi^{2}\right)$.

Observe, that for any graph $G$ of highway dimension $h d$ and maximum degree $\Delta$, we have $\kappa \leq(\Delta+1) h d$ where $\kappa$ is the skeleton dimension (Kosowski and Viennot 2017). As the graph $G_{\mathcal{I}}$ has maximum degree $\Delta=4$, it follows that the skeleton dimension of $G_{\mathcal{I}}$ is bounded by $\mathcal{O}\left(\chi^{2}\right)$.

However, with some more effort, we can show a stronger bound of $\mathcal{O}(\chi)$. We will use the following lemma, which was originally shown by Blum and Storandt (2018).

Lemma 8 (Lemma 2 in (Blum and Storandt 2018)) Consider vertices $u, v, w \in V$ such that $v$ lies on the shortest $u$-w-path. If $w$ is contained in the skeleton $T_{u}^{*}$, it is also contained in the skeleton $T_{v}^{*}$.

Proof Let $x$ be the furthest descendant of $w$ in the shortest path tree $T_{u}$ of $u$. As $w$ is contained in the shortest path three skeleton $T_{u}^{*}$, we have $\operatorname{dist}(w, x) \geq 2 \cdot \operatorname{dist}(u, w)$. Because shortest paths are unique, the node $x$ is also a descendant of $w$ in the shortest path tree $T_{v}$ of $v$ and as $\operatorname{dist}(w, x) \geq 2 \cdot \operatorname{dist}(u, w) \geq 2 \cdot \operatorname{dist}(v, w)$, the node $w$ is contained in the skeleton $T_{v}^{*}$.

The crucial property of the constructed graph is that every skeleton $T_{s}^{*}$ contains only a limited number of portal edges, as we show next. Recall that the skeleton $T_{s}^{*}$ of a shortest path tree $T_{s}$ is defined on the geometric realization, where every edge is subdivided into infinitely many infinitely short edges. We refer to vertices that were introduced during this subdivision as interior vertices. For simplicity, in the following we confuse a graph $G$ and its geometric realization $\tilde{G}$.

Lemma 9 Consider a vertex $s=v_{(a, b)}^{h}$ for $(a, b) \in[n]^{2}$ and $h \in\{1,3\}$. The skeleton $T_{s}^{*}$ contains an interior vertex of a portal edge $\left\{\rho_{\beta}^{h}, u_{\beta}^{h}\right\}$ only if we have $\beta \in\{b, b-1\}$.

Proof Assume $h=1$. For $\beta>b$, it follows from Lemma 1 that $\left\{\rho_{\beta}^{h}, u_{\beta}^{h}\right\}$ is not contained in the shortest path tree $T_{s}$ of $s$ and hence, no interior vertex of $\left\{\rho_{\beta}^{h}, u_{\beta}^{h}\right\}$ can 
be contained in $T_{s}^{*}$. Let $\beta<b-1$ and let $\rho_{\beta}^{h}=v_{(\alpha, \beta)}^{h}$. Lemma 1 implies that $u_{\beta}^{h}$ is the furthest descendant of $\rho_{\beta}^{h}$ in the shortest path tree $T_{s}$, and we have $\operatorname{dist}\left(\rho_{\beta}^{h}, u_{b}^{h}\right)<2^{\beta}$. Moreover, the distance from $v_{(a, b)}^{h}$ to $\rho_{\beta}^{h}$ is

$$
d_{(a, b)}-d_{(\alpha, \beta)}=2^{b}-2^{\beta}+\frac{a-\alpha}{n}>2^{\beta+1}>1 / 2 \cdot \operatorname{dist}\left(\rho_{\beta}^{h}, u_{b}^{h}\right) .
$$

This means that no interior vertex of $\left\{\rho_{\beta}^{h}, u_{\beta}^{h}\right\}$ can be contained in $T_{s}^{*}$. The case $h=3$ can be shown similarly.

Lemma 10 Consider a vertex $s=u_{b}^{h}$ where $b \in[n]$ and $h \in\{1,3\}$. The skeleton $T_{s}^{*}$ contains an interior vertex of a portal edge $\left\{\rho_{\beta}^{h}, u_{\beta}^{h}\right\}$ only if we have $\beta \in\{b, b-1,1\}$.

Proof Assume $h=1$. For $\beta>b$, it follows from Lemma 1 that $\left\{\rho_{\beta}^{h}, u_{\beta}^{h}\right\}$ is not contained in the shortest path tree $T_{s}$ of $s$ and hence, no interior vertex of $\left\{\rho_{\beta}^{h}, u_{\beta}^{h}\right\}$ can be contained in $T_{s}^{*}$. Let $1<\beta<b-1$ and let $\rho_{\beta}^{h}=v_{(\alpha, \beta)}^{h}$. It follows from Lemma 1 that the furthest possible descendant of $u_{\beta}^{h}$ within the shortest path tree $T_{s}$ is $v_{(1, \beta)}^{h}$, which has distance $2^{\beta}-1 / n$ from $u_{\beta}^{h}$. The distance from $u_{b}^{h}$ to $u_{\beta}^{h}$ is

$$
\operatorname{dist}\left(u_{b}^{h}, u_{\beta}^{h}\right)=2^{b}-2^{\beta}>2^{\beta+1}>\operatorname{dist}\left(u_{\beta}^{h}, v_{(1, \beta)}^{h}\right)
$$

and hence, no interior vertex of $\left\{\rho_{\beta}^{h}, u_{\beta}^{h}\right\}$ can be contained in $T_{s}^{*}$. The case $h=3$ can be shown similarly.

The previous two lemmas allow us to bound the size of any shortest path tree skeleton within a single gadget.

Lemma 11 For any $(i, j) \in[\chi]^{2}$ and any vertex s contained in $G_{i, j}$, the subtree of the skeleton $T_{s}^{*}$ induced by the vertices of $G_{i, j}$ is the union of a constant number of paths.

Proof Let $s$ be some vertex contained in some gadget $G_{i, j}$, consider the shortest path tree $T_{s}$ of $s$ and let $T_{s}\left[G_{i, j}\right]$ be the subtree of $T_{s}$ induced by the vertices of $G_{i, j}$. If we disregard all portal edges $\left\{\rho_{b}^{h}, u_{b}^{h}\right\}$, it follows from Lemma 1 that $T_{s}\left[G_{i, j}\right]$ consists of a constant number of subpaths of the cycle $O_{i, j}$, of the two paths $U_{i, j}^{1}$ and $U_{i, j}^{3}$, the 4 edges incident to $x_{i, j}^{1}, \ldots, x_{i, j}^{4}$ and some of the edges incident to $y_{i, j}$. Hence, if we do not count the portal edges, the subtree $T_{s}^{*}\left[G_{i, j}\right]$ of the skeleton $T_{s}^{*}$ induced by $G_{i, j}$ is the union of a constant number of paths. It remains to be shown that $T_{s}^{*}\left[G_{i, j}\right]$ intersects only a constant number of portal edges.

Consider first the case that $s$ is some $b$-portal, i.e., $s=\rho_{b}^{h}$ for some $h \in\{1,3\}$ and $b \in[n]$. It follows from Lemma 9 that only $\left\{\rho_{b-1}^{h}, u_{b-1}^{h}\right\}$ and $\left\{\rho_{b}^{h}, u_{b}^{h}\right\}$ can intersect $T_{s}^{*}\left[G_{i, j}\right]$. Consider now a portal edge $\left\{\rho_{\beta}^{h \boxplus 2}, u_{\beta}^{h \boxplus 2}\right\}$ on the opposite side of the cycle. It follows from Lemma 8 that it can only intersect $T_{s}^{*}\left[G_{i, j}\right]$, if it also intersects the skeleton of $\rho_{1}^{h \boxplus 2}$ or $\rho_{n}^{h \boxplus 2}$, which according to Lemma 10 holds only for $\beta \in$ $\{1, n-1, n\}$. This means that $T_{s}^{*}\left[G_{i, j}\right]$ consists of a constant number of paths. If $s$ is 
contained in some path $U^{h}$ where $h \in\{1,3\}$, we can show the same using Lemmas 8 and 10.

Assume now that $s$ is contained in the cycle $O_{i, j}$, but not a portal. Let $u$ and $v$ be the two closest portals such that $s$ is contained in the shortest $u$ - $v$-path $\pi(u, v)$. It follows from Lemma 8, that $T_{s}^{*}\left[G_{i, j}\right]$ is a subgraph of $T_{u}^{*}\left[G_{i, j}\right] \cup T_{v}^{*}\left[G_{i, j}\right] \cup \pi(u, v)$ and hence, it is the union of a constant number of paths. For similar reasons, the same holds if $s=y_{i, j}$ or $s=x_{i, j}^{h}$.

Moreover we can show that every cut in any shortest path tree skeleton of $G_{\mathcal{I}}$ intersects at most $\mathcal{O}(\chi)$ different gadgets and connecting paths between two gadgets.

Lemma 12 For every vertex $s \in V$ and every radius $r>0, \mathrm{Cut}_{r}(s)$ intersects $\mathcal{O}(\chi)$ gadgets $G_{i, j}$ and $\mathcal{O}(\chi)$ paths $P_{i, j}$ and $P_{i, j}^{\prime}$.

Proof It can be shown that for any $(i, j) \in[\chi]^{2}$, we have $\operatorname{dist}\left(y_{i, j}, y_{i+1, j}\right)=2^{n+3}+$ $4+2 / n$ and $\operatorname{dist}\left(y_{i, j}, y_{i, j+1}\right)=2^{n+3}+3+2 / n$. This means that for any $(i, j),\left(i^{\prime}, j^{\prime}\right) \in$ $[\chi]^{2}$ we have

$$
\operatorname{dist}\left(y_{i, j}, y_{i^{\prime}, j^{\prime}}\right)=\left|i-i^{\prime}\right| \cdot\left(2^{n+3}+4+2 / n\right)+\left|j-j^{\prime}\right| \cdot\left(2^{n+3}+3+2 / n\right) .
$$

Let $r>0, s \in V$ and consider a vertex $v \in \operatorname{Cut}_{r}(s)$. It holds that $\operatorname{dist}(s, v)=$ $r$. According to Lemma 2 there are two central vertices $y_{i, j}$ and $y_{i^{\prime}, j^{\prime}}$ satisfying $\operatorname{dist}\left(s, y_{i, j}\right) \leq 2^{n+2}+2^{n+1}$ and $\operatorname{dist}\left(v, y_{i^{\prime}, j^{\prime}}\right) \leq 2^{n+2}+2^{n+1}$. Using the triangle inequality we obtain that $\operatorname{dist}\left(y_{i, j}, y_{i^{\prime}, j^{\prime}}\right) \in\left[r^{-}, r^{+}\right]$where $r^{-}:=r-\left(2^{n+3}+2^{n+2}\right)$ and $r^{+}:=r+2^{n+3}+2^{n+2}$. Moreover, the ball around $y_{i^{\prime}, j^{\prime}}$ of radius $2^{n+2}+2^{n+1}$ intersects $\mathcal{O}(1)$ gadgets $G_{i^{\prime \prime}, j^{\prime \prime}}$ and $\mathcal{O}(1)$ paths $P_{i^{\prime \prime}, j^{\prime \prime}}$ and $P_{i^{\prime \prime}, j^{\prime \prime}}^{\prime}$. This means that any bound on the size of the set

$$
Y=\left\{y_{i^{\prime}, j^{\prime}} \mid \operatorname{dist}\left(y_{i, j}, y_{i^{\prime}, j^{\prime}}\right) \in\left[r^{-}, r^{+}\right]\right\}
$$

yields a bound on the number of gadgets and paths intersecting $\mathrm{Cut}_{r}(s)$.

Consider now a vertex $y_{i^{\prime}, j^{\prime}} \in Y$. Assume that $i^{\prime} \geq i$ and consider some $i^{*} \geq i^{\prime}+4$. It follows from Equation 2 and $\operatorname{dist}\left(y_{i, j}, y_{i^{\prime}, j^{\prime}}\right) \geq r^{-}$that

$$
\operatorname{dist}\left(y_{i, j}, y_{i^{*}, j^{\prime}}\right) \geq \operatorname{dist}\left(y_{i, j}, y_{i^{\prime}, j^{\prime}}\right)+4 \cdot\left(2^{n+3}+4+2 / n\right)>r^{+} .
$$

This means that $y_{i^{*}, j^{\prime}} \notin Y$ and it follows that for any $j^{\prime} \in[\chi]$ we have $\mid\left\{i^{*} \geq i \mid y_{i *}, j^{\prime} \in\right.$ $Y\} \mid \leq 3$. Similarly we can show that $\left|\left\{i^{*} \leq i \mid y_{i^{*}, j^{\prime}} \in Y\right\}\right| \leq 3$ for any $j^{\prime} \in[\chi]$. This implies $|Y| \in \mathcal{O}(\chi)$, which completes the proof.

Combining Lemmas 8, 11, and 12, we obtain that the skeleton dimension of $G_{\mathcal{I}}$ is bounded by $\mathcal{O}(\chi)$.

Lemma 13 The graph $G_{\mathcal{I}}$ has skeleton dimension $\kappa \in \mathcal{O}(\chi)$.

Proof Let $s \in V, r>0$ and consider $\operatorname{Cut}_{r}(s)$. Any vertex $v \in \operatorname{Cut}_{r}(s)$ is either contained in some gadget $G_{i, j}$ or some connecting path $P_{i, j}$ or $P_{i, j}^{\prime}$. 
We start with bounding the number of vertices that are contained in $\operatorname{Cut}_{r}(s)$ and some $P_{i, j}$ or $P_{i, j}^{\prime}$. For any $(i, j) \in[\chi]^{2}$ we have $\left|\mathrm{Cut}_{r}(s) \cap P_{i, j}\right| \leq 2$, as $P_{i, j}$ contains at most two distinct vertices that have the same distance from $s$. For the same reason we have $\left|\mathrm{Cut}_{r}(s) \cap P_{i, j}^{\prime}\right| \leq 2$. Hence, Lemma 12 implies that the size of $\operatorname{Cut}_{r}(s) \cap\left\{P_{i, j}, P_{i, j}^{\prime} \mid(i, j) \in[\chi]^{2}\right\}$ is bounded by $\mathcal{O}(\chi)$.

Consider now some gadget $G_{i, j}$. We show that $\left|\operatorname{Cut}_{r}(s) \cap G_{i, j}\right| \in \mathcal{O}(1)$. If $s$ is contained in $G_{i, j}$ this follows immediately from Lemma 11, as $\operatorname{Cut}_{r}(s)$ intersects any path in $T_{s}^{*}$ at most twice. If $s$ is not contained in $G_{i, j}$, Lemma 8 implies that $\operatorname{Cut}_{r}(s) \cap G_{i, j}$ is a subset of

$$
\left\{\mathrm{Cut}_{x_{i, j}^{h}}^{r(h)} \mid h \in[4] \text { and } r(h):=r-\operatorname{dist}\left(s, x_{i, j}^{h}\right)\right\} \cap G_{i, j} .
$$

Observe that every $x_{i, j}^{h}$ is contained in $G_{i, j}$, which means that $\left|\mathrm{Cut}_{x_{i, j}^{h}}^{r(h)} \cap G_{i, j}\right| \in \mathcal{O}(1)$. This means that the size of $\operatorname{Cut}_{r}(s) \cap\left\{G_{i, j} \mid(i, j) \in[\chi]^{2}\right\}$ is bounded by $\mathcal{O}(\chi)$. Hence we have $\left|\mathrm{Cut}_{r}(s)\right| \in \mathcal{O}(\chi)$ and it follows that $G_{\mathcal{I}}$ has skeleton dimension $\kappa \in$ $\mathcal{O}(\chi)$.

Finally we bound the pathwidth of the graph $G_{\mathcal{I}}$.

Lemma 14 The graph $G_{\mathcal{I}}$ has pathwidth $p w \in \mathcal{O}(\chi)$.

Proof Consider the graph $\hat{G}_{\mathcal{I}}$ that arises when we contract ${ }^{4}$ all vertices of degree 2 except the vertices $x_{i, j}^{h}$. It is a well-known fact that for any graph of pathwidth at least 3 , contracting a vertex of degree 2 does not decrease the pathwidth. Hence it suffices to show that $\hat{G}_{\mathcal{I}}$ has pathwidth at most $\mathcal{O}(\chi)$. For $(i, j) \in[\chi]^{2}$ denote the gadget $G_{i, j}$ and the cycle $O_{i, j}$ after the contraction by $\hat{G}_{i, j}$ and $\hat{O}_{i, j}$, respectively. We first construct a path decomposition of constant width for every $\hat{G}_{i, j}$. To this end, consider the cycle $\hat{O}_{i, j}$, which (as every cycle) has a path decomposition where every bag has size at most 3 . For $h \in\{1,3\}$ and $b \in[n]$, add $u_{b}^{h}$ to every bag containing the portal $\rho_{b}^{h}$. Finally, add $y_{i, j}$ and $x_{i, j}^{1}, \ldots, x_{i, j}^{4}$ to every bag. This yields a path decomposition of $\hat{G}_{i, j}$ which has constant width.

We now combine the path decompositions of the gadgets $\hat{G}_{i, j}$ to a path decomposition of $\hat{G}_{\mathcal{I}}$. For $(i, j) \in[\chi]^{2}$, consider the path decomposition of $\hat{G}_{i, j}$ and add the vertices $\left\{x_{i^{\prime}, j^{\prime}}^{1}, \ldots x_{i^{\prime}, j^{\prime}}^{4} \mid 1 \leq\left(i^{\prime}-i\right) \cdot \chi+\left(j^{\prime}-j\right) \leq \chi\right\}$ to every bag. According to Fig. 1, these are the vertices $x_{i^{\prime}, j^{\prime}}^{h}$ of the $\chi$ gadgets after $\hat{G}_{i, j}$ when considering the gadgets row-wise from left to right. Denote the resulting path decomposition by $\mathcal{P}_{(i-1) \cdot \chi+j}$. We can observe, that its width is bounded $\mathcal{O}(1)+4 \chi$. Concatenating all these path decompositions as $\mathcal{P}_{1}, \mathcal{P}_{2}, \ldots, \mathcal{P}_{\chi^{2}}$ then yields a path decomposition of $\hat{G}_{\mathcal{I}}$ of width $\mathcal{O}(1)+4 \chi$, which concludes the proof.

\footnotetext{
${ }^{4}$ When contracting a vertex $v$, we remove it from the graph and connect the neighbors of $v$ to a clique.
} 


\section{Conclusion}

The properties shown in the previous section now imply Theorem 1. Our reduction uses $k=5 \chi^{2}$ center vertices and the graph $G_{\mathcal{I}}$ has highway dimension $h d \in \mathcal{O}\left(\chi^{2}\right)$, skeleton dimension $\kappa \in \mathcal{O}(\chi)$ and $p w \in \mathcal{O}(\chi)$. As the $\mathrm{GT}_{\leq}$problem is $\mathrm{W}[1]$-hard for parameter $\chi$, it follows that on planar graphs of constant doubling dimension, $k$ CENTER is W[1]-hard for the combined parameter $(k, p w, h d, \kappa)$. Assuming ETH there is no $f(\chi) \cdot n^{o(\chi)}$ time algorithm for $\mathrm{GT}_{\leq}$and hence, $k$-CENTER has no $f(k, h d, p w, \kappa) \cdot|V|^{o(p w+\kappa+\sqrt{k+h d})}$ time algorithm unless ETH fails.

It follows that on planar graphs of constant doubling dimension, $k$-CENTER has no fixed-parameter algorithm for parameter $(k, p w, h d, \kappa)$ unless FPT $=\mathrm{W}[1]$. This means that we need to study approximation algorithms when parameterizing by any of the four parameters $k, p w, h d$, and $\kappa$.

It is known that classic approximation algorithms for $k$-CENTER cannot achieve any approximation factor better than 2 , unless $P=N P$. This holds even for graphs of highway dimension $h d \in \mathcal{O}\left(\log ^{2}|V|\right)$ (Feldmann 2019) or skeleton dimension $\kappa \in \mathcal{O}\left(\log ^{2}|V|\right)$ (Blum 2019). However, combining the numbers of centers $k$ with the highway dimension or the skeleton dimension allows better approximation factors than 2 in fixed-parameter time: There are EPASs for the combined parameter $(k, h d)$ (Becker et al. 2018) and the combined parameter $(k, d)$, where $d$ is the doubling dimension (Feldmann and Marx 2020). As the doubling dimension $d$ is bounded by $\mathcal{O}(\kappa)$, the latter result implies the existence of an EPAS parameterized by $k$ and the skeleton dimension $\kappa$.

Still, parameterizing solely by the number of centers $k$ does not help to overcome the general inapproximability, as there is no $(2-\epsilon)$-approximation algorithm with runtime $f(k) \cdot n^{\mathcal{O}(1)}$ for any $\epsilon>0$ and computable $f$ unless FPT $=\mathrm{W}[2]$ (Feldmann and Marx 2020). It is not known whether there is a parameterized (2- $\epsilon$ )-approximation algorithm depending only on the highway dimension $h d$ or the skeleton dimension $\kappa$, but it was shown that any such algorithm cannot run in time $2^{2^{o(\sqrt{h d})}} \cdot n^{\mathcal{O}(1)}$ or $2^{2^{o(\sqrt{\kappa})}} \cdot n^{\mathcal{O}(1)}$, respectively, unless ETH fails (Feldmann 2019; Blum 2019).

Funding Open Access funding enabled and organized by Projekt DEAL.

Open Access This article is licensed under a Creative Commons Attribution 4.0 International License, which permits use, sharing, adaptation, distribution and reproduction in any medium or format, as long as you give appropriate credit to the original author(s) and the source, provide a link to the Creative Commons licence, and indicate if changes were made. The images or other third party material in this article are included in the article's Creative Commons licence, unless indicated otherwise in a credit line to the material. If material is not included in the article's Creative Commons licence and your intended use is not permitted by statutory regulation or exceeds the permitted use, you will need to obtain permission directly from the copyright holder. To view a copy of this licence, visit http://creativecommons.org/licenses/by/4.0/.

\section{References}

Abraham I, Delling D, Fiat A, Goldberg AV, Werneck RF (2016) Highway dimension and provably efficient shortest path algorithms. J ACM. https://doi.org/10.1145/2985473 
Abraham I, Delling D, Fiat A, Goldberg AV, Werneck RFF (2011) Vc-dimension and shortest path algorithms. In: L. Aceto, M. Henzinger, J. Sgall (eds.) Proceedings of the 38th International Colloquium on Automata, Languages and Programming (ICALP'11), Lecture Notes in Computer Science, vol. 6755, pp. 690-699. Springer . https://doi.org/10.1007/978-3-642-22006-7_58

Abraham I, Fiat A, Goldberg AV, Werneck RFF (2010) Highway dimension, shortest paths, and provably efficient algorithms. In: M. Charikar (ed.) Proceedings of the 21st ACM-SIAM Symposium on Discrete Algorithms (SODA '10), pp. 782-793. SIAM . https://doi.org/10.1137/1.9781611973075.64

Becker A, Klein PN, Saulpic D (2018) Polynomial-time approximation schemes for k-center, k-median, and capacitated vehicle routing in bounded highway dimension. In: Y. Azar, H. Bast, G. Herman (eds.) Proceedings of the 26th Annual European Symposium on Algorithms (ESA '18), LIPIcs, vol. 112, pp. 8:1-8:15. Schloss Dagstuhl - Leibniz-Zentrum für Informatik . https://doi.org/10.4230/LIPIcs.ESA. 2018.8

Blum J (2019) Hierarchy of transportation network parameters and hardness results. In: B.M.P. Jansen, J.A. Telle (eds.) Proceedings of the 14th International Symposium on Parameterized and Exact Computation (IPEC '19), LIPIcs, vol. 148, pp. 4:1-4:15. Schloss Dagstuhl - Leibniz-Zentrum für Informatik . https:// doi.org/10.4230/LIPIcs.IPEC.2019.4

Blum J, Storandt S (2018) Scalability of route planning techniques. In: M. de Weerdt, S. Koenig, G. Röger, M.T.J. Spaan (eds.) Proceedings of the 28th International Conference on Automated Planning and Scheduling (ICAPS '18), pp. 20-28. AAAI Press . https://aaai.org/ocs/index.php/ICAPS/ICAPS18/ paper/view/17741

Cygan M, Fomin FV, Kowalik L, Lokshtanov D, Marx D, Pilipczuk M, Pilipczuk M, Saurabh S (2015) Parameterized Algorithms. Springer . https://doi.org/10.1007/978-3-319-21275-3

Feder T, Greene DH (1988) Optimal algorithms for approximate clustering. In: J. Simon (ed.) Proceedings of the 20th Annual ACM Symposium on Theory of Computing (STOC '88), pp. 434-444. ACM . https://doi.org/10.1145/62212.62255

Feldmann AE (2019) Fixed-parameter approximations for k-center problems in low highway dimension graphs. Algorithmica 81(3):1031-1052. https://doi.org/10.1007/s00453-018-0455-0

Feldmann AE, Marx D (2020) The parameterized hardness of the k-center problem in transportation networks. Algorithmica 82(7):1989-2005. https://doi.org/10.1007/s00453-020-00683-w

Hochbaum DS, Shmoys DB (1986) A unified approach to approximation algorithms for bottleneck problems. J ACM 33(3):533-550. https://doi.org/10.1145/5925.5933

Kosowski A, Viennot L (2017) Beyond highway dimension: Small distance labels using tree skeletons. In: P.N. Klein (ed.) Proceedings of the 28th Annual ACM-SIAM Symposium on Discrete Algorithms (SODA '17), pp. 1462-1478. SIAM . https://doi.org/10.1137/1.9781611974782.95

Plesník J (1980) On the computational complexity of centers locating in a graph. Aplikace matematiky 25(6), 445-452 . https://dml.cz/bitstream/handle/10338.dmlcz/103883/AplMat_25-1980-6_8.pdf

Publisher's Note Springer Nature remains neutral with regard to jurisdictional claims in published maps and institutional affiliations. 